\title{
MODELING OF GRAIN RAW STUFF GRINDING PROCESS
}

\author{
Marina Chkalova, Victoria Pavlidis \\ Orenburg State Agrarian University, Russia \\ chkalovamv@mail.ru,pavlidis@mail.ru
}

\begin{abstract}
The aim of the authors of the research work is to develop and build a stochastic model of the actual process of grinding feed raw materials, taking into account the zoning of the grinder working chamber. The modeling is based on the means of the correlation random functions theory, the theory of automatic control, engineering applications of operational calculus and Markov processes. As a result, the authors have developed and verified a stochastic model of the air-product layer action in the conditional zones of the working chamber and at the transition boundaries of conditional zones in the form of transfer and correlation functions, taking into account the diversity of factors influencing the efficiency of the feed raw stuff grinding process. Experimental studies have confirmed a fairly good adequacy of the models to the real process with optimal loading of the grinding chamber. During the modeling, random processes in the working chamber of the grinder are classified as Markov processes of reproduction (grinding, reducing the size of grains to the required size) and death (regrinding, walk through the holes of the sieve). The authors' method of determining the parameters of the airproduct layer in the conditional zones of the grinder working chamber and evaluating the boundary effects of transient processes has been many times used in the modeling of technological processes and feed production systems. Checking the model for adequacy to the real process was carried out in a series of laboratory and production experiments, the results of which formed the basis of engineering and technical solutions to the problem of grinding efficiency in feed preparation. Experimental samples of grinders (closed-type hammer mills with sieves) with structural changes, fixed by the Russian Federation patents, have been tested and successfully operated in the farms of the Orenburg region.
\end{abstract}

Keywords: grinder, feed, air-product layer, random process, transfer function, linear dynamical system.

\section{Introduction}

Reduction of energy intensity of technological processes and development of innovative methods of resource saving remain the most important problems of modern agricultural production. The problem is highly relevant because of contradictions between traditional technologies and the need for their modernization, between the applied methods in the field of improving the efficiency of technological processes and innovative development requirements, between the existing theoretical justifications and modern requirements of community, universality and information capacity of actual processes models.

The above fully applies to one of the most complex processes of agricultural production: the process of forage preparation and its component-grinding of grain raw stuff. Current trends in the description of the process of grinding feed are based on the principles set forth in the works of Kiskalt, Rittinger, Kick, Rebinder, Bond and many other researchers. We identified four main approaches to solving the problem of modeling of feed raw stuff grinding process, after comparative analysis of the currently available theoretical justifications of the grinding process [1-4] and in other sources.

Brief overview shows that mostly APL (air-product layer) is considered either as a stream of discrete particles without taking into account their interaction, which requires classifying and distributing a large number of influencing factors in levels of importance, or as a continuous stream, but without paying attention to grinding features of separate grains. The modern ideology of actual process modeling allows solving the problem of natural-science dualism in the framework of stochastic modeling and gives access to new engineering solutions based on the process control capabilities [4].

\section{Materials and methods}

Let us build a stochastic model of the grain raw stuff grinding process in consideration with the zoning of the grinding chamber for improving of energy and technological performance of the process by aligning the characteristics of the air-product layer.

Studies of the internal structure of the grain raw stuff grinding process confirm the unevenness of the air-product layer (APL) in different parts of the working chamber of a standard grinder (closed- 
type hammer mill) [1-7]. Our comprehensive analysis of APL action and factors affecting the efficiency of the grinding process allowed us to hypothesize the presence of conditional zones of relative uniformity of the layer and relative stability of its characteristics inside the working chamber of the grinder $[8 ; 9]$.

We show the conditional zones of the hammer mill working chamber on development drawing (Figure 1).

\begin{tabular}{l|l|l|l|}
\hline APL & \multicolumn{1}{c}{$\longrightarrow$} & \\
\hline & & & \\
\hline$a$-inlet neck & $\beta_{1}$-lower deck & $\gamma$ - sieve & $\beta_{2}$-upper deck \\
\hline
\end{tabular}

Fig.1. Conditional zones (development drawing)

The process of reproduction (grinding) and death (re-grinding, walkthrough the sieve) of some initial combination particles (grains) in the working chamber, hereinafter referred to as TD (technical device), under the steady-state mode of operation, we will consider as continuous random and denote $X(t)$. The combination of particles, able to reproduction and death, we will as denote system $S$. Under the $i$-th state of the system $S_{i}$ we will understand the quantity of particles produced at the certain moment of time (according to the certain state), where we can find ready for reproduction and death ones. There is every reason to take the random process $X(t)$ for the Markov process of death and reproduction with discrete states and continuous time [8;9]. TD is in a steady- state mode that the system states, although changing randomly, but the probabilities $p_{i}(t)(i=1,2 \ldots)$ remain constant $p_{i}(t)=P\left\{S(t)=s_{i}\right\}:$ where $S(t)-$ a random state of the system at time $t$, that is, the process consists in the transition of the system from one state to another.

We construct a marked graph of the reproduction-death process states (Figure 2), where $\lambda_{i}(t)-$ flow intensities of events leading to an increase of the random function $X(t)$ (reproduction flows), $\mu_{i}(t)$ - flow intensities of events leading to a decrease of the random function $X(t)$ (death flows).

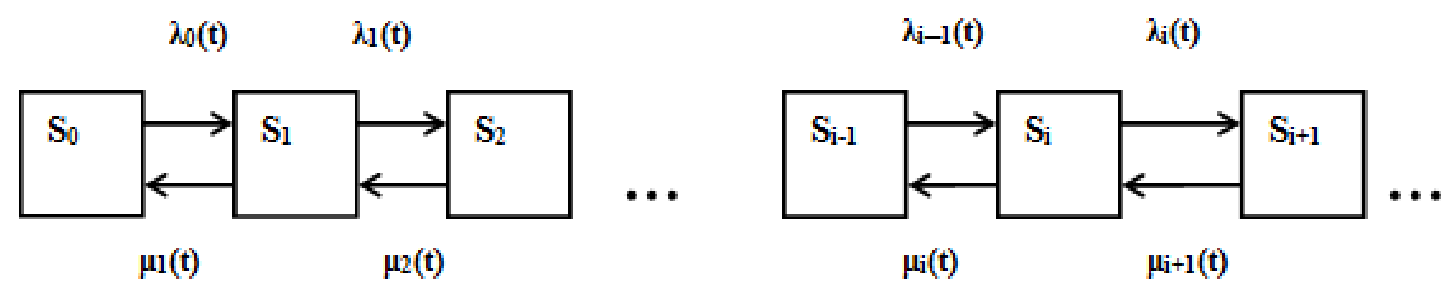

Fig. 2. Graph of process states $X(t)$

Flows of reproduced and death particles will be considered as the simplest, having the properties of stationarity, ordinariness and lack of aftereffect.

The APL under study, passing conditional zones, undergoes significant changes. Therefore, the process of reproduction and death of APL particles in different zones will proceed with its own characteristics, which should be reflected in the mathematical model. It is experimentally established that in each conditional zone the APL acts relatively stable [1-8], therefore, it can be assumed that the intensities of reproduction and death processes within one zone are constant.

In the system of introduced assumptions the random process of death and reproduction of grinding material particles in one separate zone is classified as Markov process with discrete states, continuous time and a finite number of states. There is every reason for this, since the time of APL passage through one conditional zone is very small, and the number of system states is large (the time of one APL turnover has the value about 1/3000 minutes, therefore, the time of passage through the arbitrary zone is obviously less than the indicated value). In the steady-state mode of TD operation, the investigated random process $X(t)$ is stationary and has an ergodic property (there are no states without output and without input). 
For any state of an ergodic system, the sum of all incoming probability flows is equal to the sum of all outgoing flows. Here, the flow of probability that transfers the system from state $S_{i}$ to state $S_{j}$, we understand as an expression of the form $p_{i}(t) \cdot \lambda_{i}(t)$ or $p_{i}(t) \cdot \mu_{i}(t)$, where $\lambda_{\mathrm{i}}$-intensities of reproduction flows, $\mu_{i}$-intensities of death flows $(i=0,1,2, . . n)$.

The one-dimensional distribution law of the process $X(t)$ is described by the Kolmogorov equation system corresponding to the graph [10] (Figure 2).

$$
\left\{\begin{array}{l}
\frac{d p_{0}(t)}{d t}=\mu_{1}(t) p_{1}(t)-\lambda_{1}(t) p_{0}(t) \\
\cdots \\
\frac{d p_{i}(t)}{d t}=\lambda_{i-1} p_{i-1}(t)+\mu_{i+1}(t) p_{i+1}(t)-\left(\lambda_{i}(t)+\mu_{i}(t)\right) p_{i}(t)(i=1,2,3 \ldots)
\end{array},\right.
$$

where $p_{i}(t)=P\left\{S(t)=s_{i}\right\}=P\{X(t)=i\}$ and $\lambda_{i}$ - intensities of reproduction flows; $\mu_{i}$-intensities of death flows $(i=0,1,2, .$.$) .$

Suppose $i=0,1,2, \ldots, n, \ldots$, then we have:

$$
i \cdot d p i(t) / d t=i \cdot \lambda_{i-1} \cdot p_{i-1}(t)+i \cdot \mu_{i+1}(t) \cdot p_{i+1}(t)-i \cdot\left(\lambda_{i}(t)+\mu_{i}(t)\right) \cdot p_{i}(t), i>0
$$

Let us sum up all the left and right parts:

$$
\sum_{i=1}^{\infty} i \frac{d p i(t)}{d t}=\sum_{i=1}^{\infty}\left[i \lambda_{i-1}(t) \cdot p_{i-1}(t)+i \mu_{i+1}(t) \cdot p_{i+1}(t)-i\left(\lambda_{i}(t)+\mu_{i}(t)\right) p_{i}(t)\right]
$$

Write down the left part:

$$
\sum_{i=1}^{\infty} i \cdot d p i(t) / d t=\frac{d}{d t} \cdot \sum_{i=1}^{\infty} i p_{i}(t)=\frac{d}{d t} \cdot m_{x}(t)
$$

Transforming the right part, we get:

$$
\begin{gathered}
\sum_{i=1}^{\infty} i \cdot \lambda_{i-1}(t) p_{i-1}(t)-\sum_{i=1}^{\infty} i \cdot \lambda_{i}(t) p_{i}(t)+\sum_{i=1}^{\infty} i \cdot \mu_{i+1}(t) p_{i+1}(t)-\sum_{i=1}^{\infty} i \cdot \mu_{i}(t) p_{i}(t)= \\
=\sum_{i=0}^{\infty} \lambda_{i}(t) p_{i}(t)-\sum_{i=0}^{\infty} i \lambda_{i}(t) p_{i}(t)+\sum_{i=1}^{\infty}(i-1) \mu_{i}(t) p_{i}(t)-\sum_{i=1}^{\infty} i \mu_{i}(t) p_{i}(t)= \\
=\sum_{i=0}^{\infty}(i+1-i) \cdot \lambda_{i}(t) p_{i}(t)+\sum_{i=1}^{\infty}\left((i-1-i) \cdot \mu_{i}(t) p_{i}(t)\right)=\sum_{i=0}^{\infty}\left(\lambda_{i}(t)-\mu_{i}(t)\right) p_{i}(t) \\
d m_{x}(t) / d t=\sum_{i=0}^{\infty}\left(\lambda_{i}(t)-\mu_{i}(t)\right) p_{i}(t)
\end{gathered}
$$

Since in a particular conditional zone:

$$
\begin{gathered}
\lambda_{i}(t)=\lambda(t) ; \mu_{i}(t)=\mu(t), \text { then } \\
d m_{x}(t) / d t=\sum_{i=0}^{\infty}(\lambda(t)-i \mu(t)) p_{i}(t)=\lambda(t)-\mu(t) \cdot \sum_{i=1}^{\infty} i \cdot p_{i}(t)=\lambda(t)-\mu(t) \cdot m_{x}(t) \\
d m_{x}(t) / d t=\lambda(t)-\mu(t) \cdot m_{x}(t)-\text { differential equation of order I. }
\end{gathered}
$$

Assuming, on the basis of the above reasoning, in each conditional zone: $\lambda=$ const; $\mu=$ const, we obtain an ordinary differential equation of order I, linear regarding $m_{x}(t)$ and $t$ :

$$
d m_{x}(t) / d t=\lambda-\mu \cdot m_{x}(t) \text { or } d m_{x}(t) / d t+\mu m_{x}(t)=\lambda .
$$

Solving it, we get: $m_{x}(t)=\lambda / \mu$. Reasoning similarly, we find: $D_{x}=\lambda / \mu$.

Since we have obtained $m_{x}=D_{x}=\lambda / \mu$, we can state that in the stationary mode the onedimensional distribution law of a random process in a separate zone is Poisson's law with the 
parameter $a=\lambda \mu p i=a^{i} / i ! \cdot e^{-a}$, that is, we have shown that the number of particles in any volume of the circulating layer obeys Poisson's law.

Having been able to identify a random process in a particular zone, we proceed to the description of boundary effects. We divide the general random process of death and reproduction $X(t)$ into components, corresponding to the conditional zones $X_{1}(t), X_{2}(t), X_{3}(t), X_{4}(t)$ (Figure 3 ).

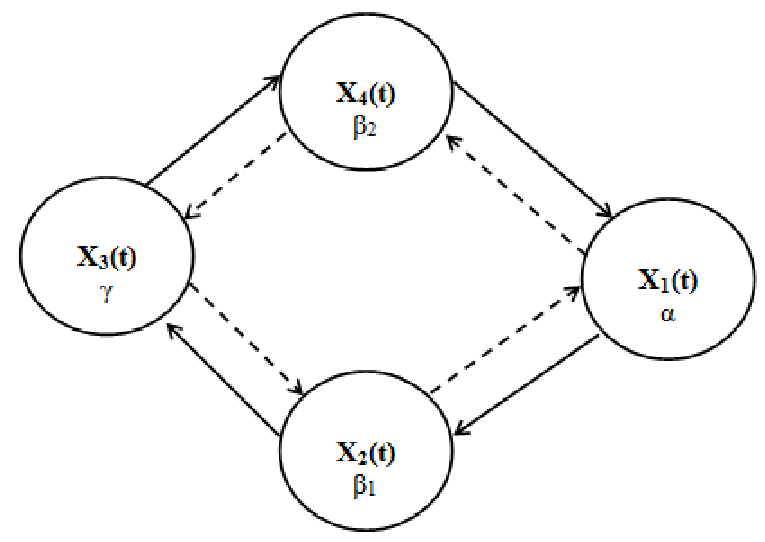

\section{Fig. 3. Dependency graph of random processes of death and reproduction corresponding to conditional zones}

The practice of stochastic modeling of real processes allows us to determine the probabilistic characteristics of the random process of death and reproduction $X_{i}(t)(i=1,2,3,4)$ using three groups of non-random parameters:

1. the number of possible states $n_{i}+1$ (since the process under study is a Markov process with discrete states);

2. intensities of reproduction flows $\lambda_{k}{ }^{i}\left(k=0,1, \ldots n_{\mathrm{i}}\right)$;

3. intensities of death flows $\mu_{k}^{i}\left(k=0,1, \ldots n_{\mathrm{i}}\right)$.

As a result, the random process $X(t)$, which components are the processes $X_{i}(t)(i=1,2,3,4)$, can be considered transitive (any of the components will be both controlling and controlled by the death and reproduction process). At the same time the process parameters $X_{2}(t) \sim\left(\lambda_{k}^{2} ; \mu_{k}^{2} ; n_{2}\right)$ will depend on the type and value of the process parameters $X_{1}(t) \sim\left(\lambda_{k}{ }^{1} ; \mu_{k}{ }^{1} ; n_{1}\right)$. We consider that the inverse influence, indicated in the graph (Figure 3) by dotted arrow, can be neglected. Process parameters of $X_{3}(t) \sim\left(\lambda_{k}^{3} ; \mu_{k}^{3} ; n_{3}\right)$ depend on types and values of the process parameters of $X_{2}(t) \sim\left(\lambda_{k}^{2} ; \mu_{k}^{2} ; n_{2}\right)$ etc.

The process considered by us in each zone is stationary in a broad sense, i.e. its probabilistic characteristics do not depend on time. In addition, each random process $X_{i}(t)(i=1,2,3,4)$ has an ergodic property, since it proceeds uniformly and the set of states is finite. Therefore, any process implementation of sufficiently long duration will " well " represent the entire possible set of implementations. We define the probabilistic characteristics of the ergodic stationary random process $X_{i}(t)$ as follows [10]:

$$
\begin{gathered}
m_{x}^{i}=\lim _{T \rightarrow \infty} \frac{1}{2 T} \int_{-T}^{T} X_{i}(t) d t \\
D_{x}^{i}=\lim _{T \rightarrow \infty} \frac{1}{2 T} \int_{-T}^{T}\left(X_{i}(t)-m_{x}^{i}\right)^{2} d t \\
k_{x}^{i}(\tau)=\lim _{T \rightarrow \infty} \frac{1}{2 T} \int_{-T}^{T}\left(X_{i}(t)-m_{x}^{i}\right)\left(X_{i}(t-\tau)-m_{x}^{i}\right) d t
\end{gathered}
$$

We write down the correlation function of the studied process using the density of dispersion distribution over the frequencies of the continuous spectrum:

$$
k_{x}^{i}(\tau)=\lim _{\Delta \omega \rightarrow 0} \sum_{k=0}^{\infty} \frac{D_{k}}{\Delta \omega} \cdot(\cos k \Delta \omega \tau) \cdot \Delta \omega=\int_{0}^{\infty} S_{x}(\omega) \cos \omega \tau \cdot d \omega,
$$




$$
\text { where } \frac{D_{k}}{\Delta \omega}=\frac{D_{k}}{\omega_{1}}=S_{x}\left(\omega_{k}\right), \lim _{\omega \rightarrow 0} \frac{D_{k}}{\Delta \omega}=S_{x}(\omega),
$$

and let us go to the spectral density via cosine by Fourier transformation:

$$
S_{x}^{i}(\omega)=\frac{2}{\pi} \int_{0}^{\infty} k_{x}(\tau) \cos \omega \tau \cdot d \tau
$$

Based on the reasoning, we build a mathematical model of the grain raw stuff grinding process. Consider two conditional zones separated by a boundary. The transition of APL through the boundary entails a change in the probabilistic characteristics of the random process $X_{i}(t)(i=1,2,3,4)$. A sufficiently small neighborhood of the boundary will be considered as a dynamic system, the input of which is a random process $X_{i}(t)$, and the output is a random process $X_{i}(t)$ (Figure 4).

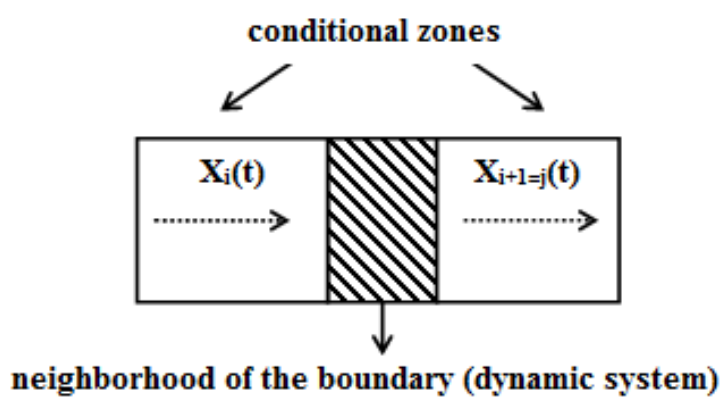

Fig. 4. Transformation of a stationary random process by a stationary dynamical system

The mathematical model of a dynamic system should be understood as a set of four elements: the state space, the space of input signals, the space of output signals and the relations linking the input, output signals and the state vector of the system (the whole set of variable states of the system). Speaking about the characteristics of the system, always have in mind the characteristics of its mathematical model, since the state of any system, its impact on the environment, all external effects on it cannot be described by any observable and even more finite set of values. The main characteristic of the system is its operator, which determines the mechanism for generating an output signal from a given input signal. The important characteristic of the quality of the system is stability, i.e. the ability of the system to return to its original state after removal of disturbing influences that have changed the amplitude and shape of the output signal. There is every reason to take the considered neighborhood of the boundary between conditional zones as a linear dynamic stationary asymptotically stable system.

In practice, the random process $X_{i}(t)$ is replaced by its implementation $x_{i}(t)$, and the random process $X_{j}(t)$ respectively by the implementation $x_{j}(t)$, with $x_{j}(t)=x_{j c}(t)+x_{j e}(t)$. With time, the natural oscillations $x_{j c}(t)$ of a stationary linear system decay, so we can consider only the second component $x_{j e}(t)$, which describes the forced oscillations under the influence of the input implementation $x_{j}(t)$. With this in mind, the harmonic oscillation of the output signal will be determined by the formula, $x_{j}(t)=G(i \omega) e^{i \omega \cdot t}$, where $G(i \omega)$ is the transfer function of a stationary linear system.

Thus, it is sufficient to define estimates

$$
\tilde{m}_{x}^{i}, \tilde{k}_{x}^{i}(\tau), \tilde{s}_{x}^{* i}(\omega), \tilde{m}_{x}^{j}, \tilde{k}_{x}^{j}(\tau), \tilde{s}_{x}^{* j}(\omega)
$$

as a result of statistical processing of the corresponding implementations of $x_{i}(t)$ and $x_{j}(t)$ of random processes $X_{i}(t)$ and $X_{i}(t)$ at the input and output of a stationary linear dynamic system (boundary with neighborhood). Assuming the obtained estimates are approximately equal to the probabilistic characteristics, we find the transfer functions of each dynamic system 


$$
\left\{\begin{array}{l}
G(i \omega)_{1 \rightarrow 2} \\
G(i \omega)_{2 \rightarrow 3} \\
G(i \omega)_{3 \rightarrow 4} \\
G(i \omega)_{4 \rightarrow 1}
\end{array}\right.
$$

The system of the transfer functions found determines the mathematical model of the grinding process under study. Models of processes in conditional zones will be correlation functions

$$
\tilde{k}_{x}^{1}(\tau), \tilde{k}_{x}^{2}(\tau), \tilde{k}_{x}^{3}(\tau), \tilde{k}_{x}^{4}(\tau) .
$$

\section{Results and discussion}

We will use mathematical expressions of characteristics (2), but we will transform them a little for convenience of practical application. Replace the integral

$$
m_{x}^{i}=\frac{1}{T} \int_{0}^{T} x(t) d t
$$

with a finite sum. To do this, divide the time interval into $n$ equal segments with the length $\Delta t$. The midpoints of segments denote $t_{1}, t_{2}, \ldots t_{n}$. Let us represent the initial integral as sum of integrals over elementary segments $\Delta t$, we replace the integrand function on each segment by the mean value corresponding to the center of the segment.

$$
m_{x}^{i}=\frac{1}{T} \cdot \frac{T}{n} \cdot \sum_{i=1}^{n} x\left(t_{i}\right)=\frac{1}{n} \cdot \sum_{i=1}^{n} x\left(t_{i}\right)
$$

with

$$
D_{x}^{i}=\frac{\sum_{i=1}^{n}\left(x\left(t_{i}\right)\right)^{2}}{n} ; \sigma_{x}^{i}=\sqrt{D_{x}^{i}}
$$

Denote $\tau=m \cdot \Delta t=\mathrm{m} \cdot T / n$, where $m$ - the parts quantity of segment $\Delta t$ time $T$ and receive expression for the correlation function:

$$
k_{x}^{i}(\tau)=\frac{\sum_{i=1}^{n-m} x\left(t_{i}\right) \cdot x\left(t_{i+m}\right)}{n-m} .
$$

Normalizing the correlation function, we get $\rho_{x}^{i}(\tau)$. We approximate, thus smooth out the irregular oscillations of the experimentally found function by means of the theoretical function $\rho_{x}{ }^{*}(\tau)$, the parameters of which are selected by the method of least squares, what allows us to obtain a normalized spectral density of the random process of death and reproduction $X_{i}(t)$

$$
s_{x}^{*_{i}}=\frac{1}{2 \pi} \int_{-\infty}^{+\infty} k_{x}^{i}(\tau) \cdot e^{-i \omega \tau} d \tau .
$$

We apply the principle of the cybernetic box [10-12] to obtain transfer functions of linear dynamical systems (neighborhoods of boundaries between conditional zones).

It is known that the spectral density of a stationary random process $X_{j}(t)$ will be equal to the spectral density of the process $X_{i}(t)$, multiplied by the square of the frequency response module (TF) [10-12]

$$
\tilde{s}_{x}^{* j}(\omega)=\left|G(i \omega)_{i \rightarrow i+1=j}\right|^{2} \cdot \tilde{s}_{x}^{* i}(\omega) .
$$

Suppose, estimates of correlation functions of input and output random processes $\tilde{k}_{x}^{i}(\tau), \tilde{k}_{x}^{j}(\tau)$ are obtained as a result of experimental data processing. On their basis, estimates of spectral densities (normalized spectral densities) and transfer functions can be found

$$
\tilde{s}_{x}^{*_{i}}(\omega), \tilde{s}_{x}^{*_{j}}(\omega),\left|G(i \omega)_{i \rightarrow i+1=j}\right|^{2}=\frac{\tilde{s}_{x}^{*_{j}}(\omega)}{\tilde{s}_{x}^{*_{i}}(\omega)} .
$$


To determine the estimates of spectral densities, we use the correspondence table of correlation functions, most often used in engineering studies, and spectral densities. We were guided by the following considerations, when selected an analytical correlation function: the function should not contain a multiplier of the form $e^{-\alpha|\tau|}$, leading to monotony; it should include both harmonics.

After a comparative analysis we gave preference to the function of the form

$$
k_{x}(\tau)=2 \alpha^{2}(2 \cos \beta \tau-1) \cdot \frac{\sin \beta \tau}{\tau},(9)
$$

with corresponded spectral density

$$
S_{x}^{*}(\omega)=\left\{\begin{array}{c}
0, \text { for } 0 \leq|\omega| \leq \beta \\
\alpha^{2}, \text { for } \beta<|\omega|<2 \beta \\
0, \text { for } 2 \beta<|\omega|
\end{array}\right.
$$

where $\alpha$ and $\beta$-parameters chosen by the least squares method.

In this case, the error of the system operation, caused by a change in the frequency of the input signal, can be determined by the formula $[11 ; 12]$

$$
\varepsilon_{x}(\omega)=1-G(i \omega)
$$

\section{Novelty of the research}

The novelty of the research conducted by the authors is as follows. The obtained model fully describes the action of APL in the working chamber of the grinder and allows us to conclude the following:

1. the number of particles in any volume of circulating APL obeys Poisson's law;

2. the neighborhood of the boundary between conditional zones is a linear dynamic stationary asymptotically stable system;

3. serial connection of stationary linear systems gives a stationary linear system, the transfer function of which is equal to the product of the transfer functions of the connected systems

$$
G(i \omega)=\Pi_{k=1}^{4} G_{k}(i \omega),
$$

and the result of such a connection does not depend on the order of connection;

4. the system of the found transfer functions

$$
\text { (7) }\left|G(i \omega)_{i \rightarrow i+1=j}\right|^{2}=\frac{\tilde{s}_{x}^{* j}(\omega)}{\tilde{s}_{x}^{* i}(\omega)}
$$

determines the global mathematical model of the grain raw stuff grinding process in the working chamber; 5) local model of APL action in conditional zones of the grinder will serve as a system of correlation functions

$$
\tilde{k}_{x}^{1}(\tau), \tilde{k}_{x}^{2}(\tau), \tilde{k}_{x}^{3}(\tau), \tilde{k}_{x}^{4}(\tau) \text { of the form } k_{x}(\tau)=2 \alpha^{2}(2 \cos \beta \tau-1) \cdot \frac{\sin \beta \tau}{\tau} .
$$

The model constructed by the authors was verified in the course of laboratory and production experiments on the basis of the Engineering Faculty and training and production facilities of the Orenburg State Agrarian University. Model verification yielded the following results (grinded material - medium dry barley) [13]:

$$
\begin{aligned}
& \text { zone } \alpha: \alpha=4.487 \cdot 10^{3}, \beta=0.692 ; \text { zone } \beta_{1}: \alpha=3.25 \cdot 10^{3}, \beta=0.655 \\
& \text { zone } \gamma: \alpha=4.787 \cdot 10^{3}, \beta=3.301 ; \text { zone } \beta_{2}: \alpha=3.676 \cdot 10^{4}, \beta=1.327
\end{aligned}
$$

For transient processes $(8,11)$ :

$$
G(i \omega)_{1 \rightarrow 2}=\frac{7.15}{7.55}, \varepsilon_{x}(\omega) \approx 0.053 ; G(i \omega)_{2 \rightarrow 3}=\frac{4.205}{4.41}, \varepsilon_{x}(\omega) \approx 0.046 ;
$$




$$
G(i \omega)_{3 \rightarrow 4}=\frac{2.815}{2.901}, \varepsilon_{x}(\omega) \approx 0.0296 ; G(i \omega)_{4 \rightarrow 1}=\frac{3.487}{3.55}, \varepsilon_{x}(\omega) \approx 0.018
$$

\section{Conclusions}

Experimental studies have confirmed a fairly good adequacy of the models to the real process with optimal loading of the grinding chamber. There were developed recommendations for improving of the working chamber design of a closed type hammer mill, which help align the characteristics of APL around the perimeter of the working chamber and "blur" the boundaries of conditional zones. The established regularities of APL action in the working chamber of the grinder, which determine the prospects for management of its parameters, formed the basis of inventions secured by patents of the Russian Federation: Grain grinder (patent No. 25687540), Piezoelectric shock sensor (patent No. 2689895), Method for controlling the granulometric composition of grinded grain material (patent No. 2688352), Method for determining the granulometric composition of grinded grain material (patent No. 2688771), Software tool for pulse signal processing of an electronic oscilloscope during grain raw material grinding (ST No. 2018611777), Program for fractional composition calculation of grain raw materials during grinding in a hammer mill (ST No. 2018618240).

Prototypes of shredders (closed-type hammer crushers with grates) with structural changes have been tested and successfully operated in the farms of the Sharlyk and Alexander districts of the Orenburg region.

\section{References}

[1] Bond F.C. Some recent advances in grinding theory and practice / Brit. Fnang., No.9,1963.pp. 84-93.

[2] Kiesskalt. Neue Ergebnisse der Feinzerkleinerung. Ver. Deut. Ing. Vol. 97, 1955.

[3] Grassman P. Energie und Exergie. - BWK, 1961, Bd. 13, No. 11, pp. 482-486.

[4] Goryachkin V. P. Collected works. - Moscow: Kolos, 1965.

[5] Melnikov S. V. Mechanization and automation of livestock farms. Leningrad: Kolos, 1978, 560 p.

[6] Kukta G. M. Machines and equipment for the preparation of feed / Moscow: Agropromizdat, 1987, $303 \mathrm{p}$.

[7] Myand A. E. Fodder-procession machines and units / Moscow: Mashinostroenie, 1970, pp. 117160.

[8] Aleshkin V. R., Roshchin P. M. Mechanization of animal husbandry: textbook. - 2nd ed., Rev. and add. / Moscow: Kolos, 1993, 319 p.

[9] Rotating circular die, Patent Ap. Pub. No.: US 2003/0091710, Int. CI.7 A21D 6/00, Rick Wendell Bajema, Pub. Date: May 15, 2003.

[10] Pavlidis V.D., Chkalova M.V. Mathematical modeling of technological processes in agricultural production / Kormoproizvodstvo: scientific and practical journal, No.10, 2008. pp. 26-28

[11] Wentzel E. S., Ovcharov L. A. Theory of random processes and its engineering applications: manual for universitiy students; ed. 3rd, rev. and add. / Moscow: Publishing House "ACADEMIA", 2003, $432 \mathrm{p}$.

[12] Pugachev V. S., Sinitsyn I. N. Theory of stochastic systems / Moscow: Logos, 2004, 1000 p.

[13] Chkalova M.V., Shakhov V. A., Burlutsky E. M., Pavlidis V.D. Determination of quantitative parameters of the APL in the working chamber of the grinder / Achievements of science and technology of the agro-industrial complex: theoretical and scientific-practical journal, No.12, 2017, pp. 57-60. 